\title{
Proteasome subunit expression analysis and chemosensitivity in relapsed paediatric acute leukaemia patients receiving bortezomib-containing chemotherapy
}

Denise Niewerth', Gertjan J. L. Kaspers ${ }^{1}$, Gerrit Jansen², Johan van Meerloo ${ }^{1,3}$, Sonja Zweegman ${ }^{3}$, Gaye Jenkins ${ }^{4}$, James A. Whitlock ${ }^{5}$, Stephen P. Hunger ${ }^{6}$, Xiaomin Lu', Todd A. Alonzo ${ }^{8}$, Peter M. van de Ven ${ }^{9}$,

Terzah M. Horton ${ }^{4}$ and Jacqueline Cloos ${ }^{1,3^{*}}$

\begin{abstract}
Background: Drug combinations of the proteasome inhibitor bortezomib with cytotoxic chemotherapy are currently evaluated in phase 2 and 3 trials for the treatment of paediatric acute myeloid leukaemia (AML) and acute lymphocytic leukaemia (ALL).

Methods: We investigated whether expression ratios of immunoproteasome to constitutive proteasome in leukaemic cells correlated with response to bortezomib-containing re-induction chemotherapy in patients with relapsed and refractory acute leukaemia, enrolled in two Children's Oncology Group phase 2 trials of bortezomib for ALL (COG-AALL07P1) and AML (COG-AAML07P1). Expression of proteasome subunits was examined in 72 patient samples (ALL $n=60$, AML $n=12$ ) obtained before start of therapy. Statistical significance between groups was determined by Mann-Whitney $U$ test.

Results: Ratios of immunoproteasome to constitutive proteasome subunit expression were significantly higher in pre-B ALL cells than in AML cells for both $\beta 5 i / \beta 5$ and $\beta 1 \mathrm{i} / \beta 1$ subunits $(p=0.004$ and $p<0.001)$. These ratios correlated with therapy response in AML patients; $\beta 1 \mathrm{i} / \beta 1$ ratios were significantly higher $(p=0.028)$ between patients who did $(n=4)$ and did not reach complete remission (CR) $(n=8)$, although for $\beta 5 i / \beta 5$ ratios, this did not reach significance. For ALL patients, the subunit ratios were also higher for patients who showed a good early response to therapy but this relation was not statistically significant. Overall, for this study, the patients were treated with combination therapy, so response was not only attributed to proteasome inhibition. Moreover, the leukaemic blast cells were not purified for these samples.
\end{abstract}

Conclusions: These first ex vivo results encourage further studies into relative proteasome subunit expression to improve proteasome inhibition-containing therapy and as a potential indicator of bortezomib response in acute leukaemia.

Keywords: Pediatric acute leukaemia, Bortezomib, Proteasome inhibitor, Immunoproteasome

Abbreviations: AML, Acute myeloid leukaemia; ALL, Acute lymphocytic leukaemia; COG, Children's Oncology Group; CR, Complete remission; MM, Multiple myeloma; PI, Proteasome inhibitor; ER, Endoplasmic reticulum; UPR, Unfolded protein response

\footnotetext{
*Correspondence: j.cloos@vumc.nl

'Department of Pediatric Oncology/Hematology, VU University Medical

Center, Amsterdam, The Netherlands

${ }^{3}$ Department of Hematology, VU University Medical Center, De Boelelaan

1117, 1081 HV Amsterdam, The Netherlands

Full list of author information is available at the end of the article
} 


\section{Background}

The survival of paediatric patients with leukaemia has greatly improved in recent decades. However, 10$30 \%$ of patients still relapse, and outcome after relapse remains poor, with remission rates as low as $10-40 \%$ for poor prognostic subtypes [1, 2]. Hence, there is a need for improvement in therapeutic options for these patients. Based on the proven clinical activity in multiple myeloma (MM) [3] and mantle cell lymphoma [4], as well as encouraging preclinical data from our lab [5] and the Paediatric Preclinical Testing Program [6], the proteasome inhibitor (PI) bortezomib is currently undergoing clinical evaluation in paediatric leukaemia $[7,8]$.

Proteasomes are large, multi-subunit complexes located in both the cytosol and nucleus and are responsible for the degradation of the majority of intracellular proteins. In addition to constitutive proteasomes $(\beta 1+$ $\beta 2+\beta 5)$, the immunoproteasome $(\beta 1 i+\beta 2 i+\beta 5 i)$ represents a proteasome variant that is predominantly expressed in haematopoietic cells [9-11]. The immunoproteasomes produce peptides similar to constitutive proteasomes. Although the exact role of the immunoproteasome has yet to be defined, the peptide fragments produced by the immunoproteasome are thought to play a role in antigen presentation to $\mathrm{MHC}$ class I antigens (reviewed in [12]). Disruption of proteasome activity results in rapid accumulation of toxic regulatory proteins in the cell that results in endoplasmic reticulum (ER) stress and activates the unfolded protein response (UPR), which blocks protein translation and initiates alternative degradation pathways in stress situations [13]. Another mechanism of action of bortezomib is thought to inhibit NF-kB chemotherapy-induced pro-survival pathways in leukaemic blasts by blocking the proteasomal degradation of NF-kB's natural inhibitor, IkB [14].

Although bortezomib displays only modest singleagent activity in children [15] and adults [16-18], it has additive or synergistic interactions with cytotoxic agents commonly used to treat acute leukaemia including glucocorticoids, vincristine [5] and anthracyclines [19]. Phase $1 / 2$ studies combining bortezomib with standard induction/consolidation regimens have shown promising clinical activity in both adult AML [20-22] and paediatric ALL $[8,23]$. However, bortezomib did not improve complete remission (CR) rate or overall survival in a paediatric AML cohort [7]. Thus, the clinical impact of PI therapy in combination with cytotoxic chemotherapy has not yet been fully assessed.

Despite the encouraging results of bortezomib in early-phase clinical trials, primary or acquired resistance to bortezomib may limit its efficacy [24, 25] and thus identifying those patients who respond to bortezomib-containing therapy is of great clinical relevance. Recently, we reported that higher ratios of immunoproteasome to constitutive proteasome protein expression in paediatric ALL and AML leukaemia cells at diagnosis predicted ex vivo sensitivity to bortezomib and other PIs [10]. The importance of subunit assembly for PI sensitivity was further investigated in leukaemia cell lines, revealing that interferon- $\gamma$-induced upregulation of immunoproteasome subunit expression and concomitant downregulation of constitutive subunit expression, markedly sensitizing bortezomib-resistant cells to PIs, with the $\beta 5 \mathrm{i}$ being the most important subunit involved in this process [26].

Here, we explored whether ratios of immunoproteasome to constitutive proteasome protein expression correlate with bortezomib response in paediatric leukaemia patients treated with bortezomib-containing re-induction chemotherapy. Specifically, paediatric patients with acute leukaemia in first relapse were enrolled in two Children's Oncology Group (COG) phase 2 trials of bortezomib combined with re-induction chemotherapy for paediatric ALL (AALL07P1) or paediatric AML (AAML07P1). In addition to (immuno) proteasome profiling, we also examined leukaemia cells for bortezomib-induced alterations in NF-kB activity.

\section{Methods}

\section{Patients}

Pre-treatment, snap-frozen peripheral blood mononuclear cells (PBMCs) or mononuclear cells isolated from the bone marrow were obtained from 84 patients enrolled in either the ALL (COG-AALL07P1; NCT00873093) or AML (COG-AAML07P1; NCT00666588) clinical trials between 2009 and 2013. Since no differences in overall survival between the two randomization arms in AAML07P1 were observed [7], no distinction was made between AML patients receiving etoposide or idarubicin. Informed consent was obtained from the patient or their parent(s), and the studies have been performed according to the Declaration of Helsinki. Assent, as appropriate, were obtained in accordance with the US National Cancer Institute, and the study was approved by the relevant COG committees, CTEP and the paediatric central IRB in accordance with institutional policies for human subjects' research. Mononuclear cells were isolated using Ficoll density centrifugation prior to proteasome and NF-kB analysis. Only samples with blast percentages $>20 \%$ were included in the analyses. Seventy-two patient samples were available for proteasome subunit expression analysis (60 ALL and 12 AML), and 32 patient samples (22 ALL and $10 \mathrm{AML}$ ) for proteasome subunit catalytic activity. Forty-eight patient samples (36 ALL and 12 AML) were evaluable for NF-kB analysis. Table 1 summarizes patient demographics. 
Table 1 Patient demographics

\begin{tabular}{|c|c|c|}
\hline & $\operatorname{ALL}(n=68)$ & $\operatorname{AML}(n=16)$ \\
\hline Age, years median (range) & $9.9(1.5-23.7)$ & $9.6(1.0-19.6)$ \\
\hline \multicolumn{3}{|l|}{ Sex } \\
\hline Female & 22 & 9 \\
\hline Male & 46 & 7 \\
\hline \multicolumn{3}{|l|}{ Race } \\
\hline Caucasian & 47 & 10 \\
\hline Black-American & 9 & 1 \\
\hline Asian/Indian & 5 & 4 \\
\hline Native American/Hawaiian & 1 & 0 \\
\hline Other/unknown & 6 & 1 \\
\hline \multicolumn{3}{|l|}{ Ethnicity } \\
\hline Hispanic & 21 & 3 \\
\hline Non-Hispanic & 46 & 11 \\
\hline Unknown & 1 & 2 \\
\hline \multicolumn{3}{|l|}{ Performance score (ECOG) } \\
\hline 0 & 56 & 9 \\
\hline 1 & 9 & 7 \\
\hline 2 & 3 & 0 \\
\hline WBC median, $10^{9} / \mathrm{L}$ (range) & $12.8(0.3-201.1)$ & $11.6(0.5-61.8)$ \\
\hline \multicolumn{3}{|l|}{ ALL histologic subtype } \\
\hline pre-B ALL & 52 & \\
\hline T-ALL & 16 & \\
\hline \multicolumn{3}{|l|}{ AML type } \\
\hline Relapsed & & 11 \\
\hline Secondary & & 5 \\
\hline \multicolumn{3}{|l|}{ AML: prior BMT } \\
\hline No & & 12 \\
\hline Yes & & 4 \\
\hline
\end{tabular}

\section{Protein expression/Western blotting}

Antibodies to proteasome subunits $\beta 1, \beta 2, \beta 5, \beta 1 \mathrm{i}$ and $\beta 5 \mathrm{i}$ were purchased from Enzo Life Sciences (Farmingdale, NY, USA), and the IRDye infraredlabelled secondary antibodies were from LI-COR Biosciences (Lincoln, NE, USA). In addition, anti-actin (clone C4) was purchased from Millipore (Temecula, CA, USA). Protein expression levels of constitutive proteasome subunits $\beta 5(\mathrm{PW}-8895)$ and $\beta 1$ (PW8140 ) and immunoproteasome subunits $\beta 5 \mathrm{i}$ (PW$8845)$ and $\beta 1 \mathrm{i}(\mathrm{PW}-8345)$ were determined by Western blot analysis as previously described [10, 26, 27]. Protein bands were quantified by Odyssey software, corrected for background, and normalized with $\beta$ actin. Subunit expression between patient samples on different gels was normalized using subunit expression in the leukaemic T-ALL cell line CCRF-CEM [27].
Subunit-specific $\beta 5$ and $\beta 5$ i proteasome catalytic activities Subunit-specific proteasome catalytic activity of $\beta 5$ (AcWLA-AMC) and $\beta 5 i$ (Ac-ANW-AMC) was measured using fluorogenic substrates [28] as previously described [26] in cell extracts from 22 ALL and 10 AML samples.

\section{Active p65 NF-kB by ELISA}

PBMCs $(n=48)$ were isolated from whole blood of patients prior to bortezomib treatment and at 6 and $24 \mathrm{~h}$ after the first bortezomib dose. Nuclear lysates were prepared, and active p65 NF-kB was determined by ELISA (Active Motif, Carlsbad, CA) as previously described [15]. NF-kB ELISA activity was performed in triplicate and averages used for comparisons over time and blast percentage ranged from 21 to $93 \%$ (median $53 \%)$ for ALL $(n=36)$ and from 22 to $85 \%$ (median $52 \%)$ for AML $(n=12)$.

\section{Statistics}

Correlations were calculated by determining Spearman's rank correlation coefficients. Statistical significance between groups was determined by Mann-Whitney $U$ test. Logistic regression analysis was used to determine whether the measured predictor variables were associated with response to therapy, both with and without correction for leukaemia type (ALL/AML), blast percentages, gender, age and white blood cell count. All statistical analyses were performed using IBM-SPSS (version 20.0). A two-sided significance level of 0.05 was used in all statistical analyses.

\section{Results}

Proteasome subunit expression in relapsed childhood acute leukaemia samples

Consistent with our previous preliminary findings [10], in this cohort of 60 relapsed ALL (46 pre-B ALL, 14 T-ALL) and 12 relapsed AML patients, AML blasts did not differ from pre-B ALL or T-ALL blasts in constitutive $\beta 1$ subunit expression (Fig. 1a) while both AML blasts and TALL blasts had higher $\beta 5$ subunit expression than pre-B ALL blasts $(p=0.005$ and $p=0.041$, respectively; Fig. $1 \mathrm{~b}$; Table 2). In this subset of samples, ALL samples had a significantly higher blast percentage (median $84 \%$ ) than AML samples (median $59 \%)(p<0.001)$. In order to determine if the normal cells in the AML samples contribute to the proteasome subunit composition, proteasome subunit expression of PBMCs from healthy adult volunteers $(n=5)$ were compared to pre-B ALL and AML samples and revealed that the median constitutive $\beta 1$ and $\beta 5$ expression are both significantly lower in PBMCs compared to AML patient samples ( $\beta 1 p=0.037$ and $\beta 5 p=0.002)$, indicating that the normal cells in leukaemia samples do not significantly contribute to the proteasome subunit expression comparisons (Fig. 1a, b). In contrast, pre-B 

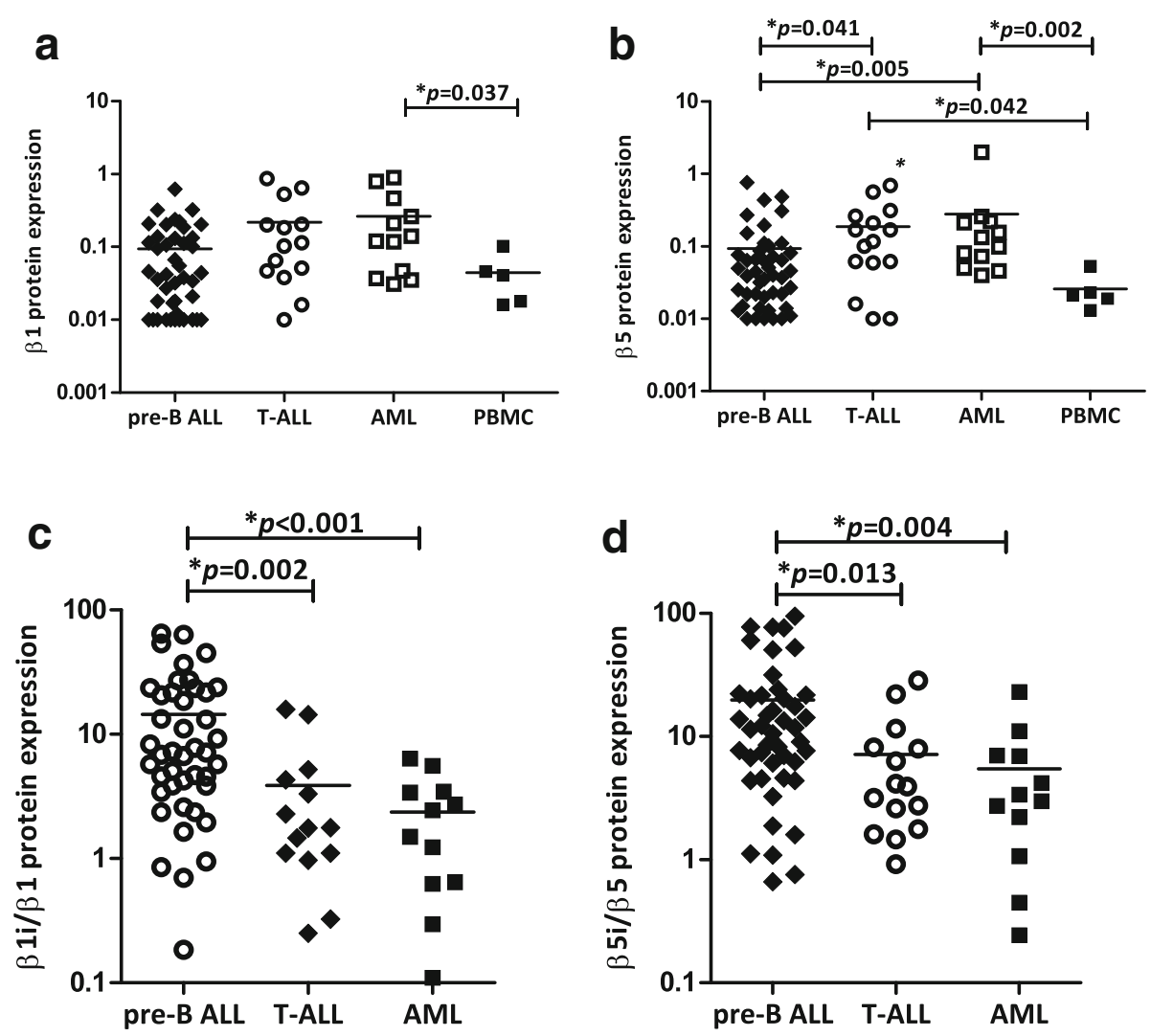

Fig. 1 Proteasome subunit protein expression in relapsed childhood ALL and AML. Comparison of a constitutive subunit $\beta 1$ b and constitutive subunit $\beta 5$ expression for pre-B ALL $(n=46)$, T-ALL $(n=14)$ and AML $(n=12)$ patient samples and PBMCs from healthy adult volunteers $(n=5)$. $\mathbf{c}$ The ratio of paired subunits $\beta 1 \mathrm{i} / \beta 1$. d The ratio of paired subunits $\beta 5 \mathrm{i} / \beta 5$, within each patient sample for pre-B ALL $(n=46)$, T-ALL $(n=14)$ and AML $(n=12)$ patient samples. Protein expression was assessed by Western blotting and expressed as relative quantifications of subunit expression (ratio proteasome subunit/ $\beta$-actin based on loading of $15 \mu \mathrm{g}$ total protein, normalized to CEM). The line denotes the mean. Significant $p$ values are noted (Mann-Whitney $U$ test)

Table 2 Median proteasome subunit protein expression and NF-kB activity in paediatric acute leukaemia patients

\begin{tabular}{cccccccc}
\hline & $\beta 5$ (range) & $\beta 1$ (range) & $\beta 5 i$ (range) & $\beta 1$ (range) & $\beta 5 i / \beta 5$ (range) & $\beta 1$ i/ $\beta 1$ (range) & NF-kB activity \\
\hline ALL & $0.055(0.01-0.76)$ & $0.05(0.01-0.86)$ & $0.46(0.06-3.43)$ & $0.24(0.07-1.45)$ & $8.2(0.66-94.5)$ & $5.2(0.18-64.7)$ & $128.1(11.8-1025.7)$ \\
CR & $0.045(0.01-0.76)$ & $0.05(0.01-0.64)$ & $0.47(0.06-2.08)$ & $0.31(0.07-1.06)$ & $8.55(0.76-94.5)$ & $6.8(0.19-64.7)$ & $253.5(11.8-1022.2)$ \\
No CR & $0.065(0.01-0.56)$ & $0.1(0.01-0.86)$ & $0.38(0.13-3.43)$ & $0.22(0.07-1.45)$ & $6.7(0.66-52.5)$ & $3.4(0.25-27.2)$ & $73.0(24.5-1025.7)$ \\
Pre-B ALL & $0.04(0.01-0.76)$ & $0.04(0.01-0.62)$ & $0.46(0.06-3.43)$ & $0.33(0.07-1.45)$ & $11.4(0.66-94.5)$ & $7.1(0.19-64.7)$ & $90.2(11.8-1025.7)$ \\
CR & $0.03(0.01-0.76)$ & $0.04(0.01-0.62)$ & $0.57(0.06-2.08)$ & $0.43(0.07-1.06)$ & $13.4(0.76-94.5)$ & $7.8(0.19-64.7)$ & $89.9(11.8-1022.2)$ \\
No CR & $0.06(0.01-0.49)$ & $0.1(0.01-0.32)$ & $0.42(0.13-3.43)$ & $0.23(0.12-1.45)$ & $9.0(0.66-52.5)$ & $4.2(0.7-27.2)$ & $98.9(24.5-1025.7)$ \\
T-ALL & $0.12(0.01-0.69)$ & $0.11(0.01-0.86)$ & $0.44(0.17-1.12)$ & $0.21(0.07-0.58)$ & $3.9(0.92-28.5)$ & $1.77(0.25-15.9)$ & $333.7(35.3-625.3)$ \\
CR & $0.21(0.06-0.56)$ & $0.1(0.04-0.86)$ & $0.37(0.17-0.82)$ & $0.11(0.07-0.21)$ & $1.78(1.46-2.74)$ & $1.1(0.25-1.77)$ & $393.5(262.5-625.3)$ \\
No CR & $0.1(0.01-0.69)$ & $0.11(0.01-0.64)$ & $0.46(0.18-1.12)$ & $0.22(0.16-0.58)$ & $5.2(0.92-28.5)$ & $2.28(0.33-15.9)$ & $37.9(35.3-40.5)$ \\
AML & $0.12(0.04-1.98)$ & $0.13(0.03-0.88)$ & $0.41(0.09-1.08)$ & $0.17(0.1-0.64)$ & $3.2(0.24-23.0)$ & $1.97(0.11-6.4)$ & $524.5(35.3-1323.7)$ \\
CR & $0.089(0.04-0.21)$ & $0.042(0.031-0.26)$ & $0.23(0.09-0.92)$ & $0.23(0.13-0.64)$ & $4.0(0.45-23.0)$ & $4.5(2.4-6.3)^{a}$ & $804.1(284.5-1323.7)$ \\
No CR & $0.13(0.05-1.98)$ & $0.18(0.04-0.88)$ & $0.52(0.22-1.1)$ & $0.16(0.1-0.49)$ & $3.2(0.24-11.1)$ & $0.94(0.11-3.5)$ & $524.5(35.3-1246.5)$ \\
\hline
\end{tabular}

Please note that Western blot data depict relative quantifications of subunit expression (ratio proteasome subunit/ $\beta$-actin based on loading of $15 \mu \mathrm{g}$ total protein, normalized to CEM). Quantifications were based on one Western blot analysis per sample. For three patients multiple replicate quantifications of subunits could be performed with a mean SD of $<26 \%$. Lower limit of detection of protein bands quantification by Odyssey software; set to $0.01 \mathrm{AU}$ for $\beta 5$ and $\beta 1,0.06 \mathrm{AU}$ for $\beta 5 \mathrm{i}$ and $0.07 \mathrm{AU}$ for $\beta 1 \mathrm{i}$

Pre-B ALL $n=45$ patient samples for $\beta 5$ and $\beta 5 \mathrm{i}, n=43$ for $\beta 1$ and $\beta 1 \mathrm{i}$, T-ALL $n=15$ for $\beta 5$ and $\beta 5 \mathrm{i}, n=14$ for $\beta 1$ and $\beta 1 \mathrm{i}$, AML $n=12$ patient samples were used $C R$ complete remission 
ALL blasts had higher immunoproteasome subunit $\beta 1 \mathrm{i}$ expression than AML blasts $(p=0.048)$, while no significant difference was observed between ALL and AML for the expression of immunoproteasome subunit $\beta 5 \mathrm{i}$ (Additional file 1: Figure S1A, B). As a consequence, median ratios of both $\beta 1 \mathrm{i} / \beta 1$ and $\beta 5 \mathrm{i} / \beta 5$ expression were significantly higher in pre-B ALL patients than in T-ALL patients $(p=0.002$ for $\beta 1 \mathrm{i} / \beta 1$ and $p=0.013$ for $\beta 5 \mathrm{i} / \beta 5)$ and in AML patients $(p<0.001$ for $\beta 1 \mathrm{i} / \beta 1$ and $p=0.004$ for $\beta 5 \mathrm{i} / \beta 5$ Fig. $1 \mathrm{c}$, $d$; Table 2). Median blast percentages did not differ between T-ALL (median $81 \%$ ) and pre-B ALL (median $86 \%$ ).
Proteasome subunit expression correlations with response to re-induction therapy

Next, we compared differences in proteasome protein subunit expression between patients that attained CR vs. no $C R$ after the first re-induction therapy block. When samples were subdivided by leukaemia subtype, a statistically significant difference was detected in median $\beta 1 \mathrm{i} /$ $\beta 1$ ratios between CR $(n=4)$ and no CR $(n=8)$ in patients with AML $(p=0.028$, Fig. 2a; Table 2$)$. No significant differences were detected for median $\beta 5 i / \beta 5$ ratios, also not within ALL subgroups (Fig. 2b, c). Median blast percentages did not differ between patients that attained
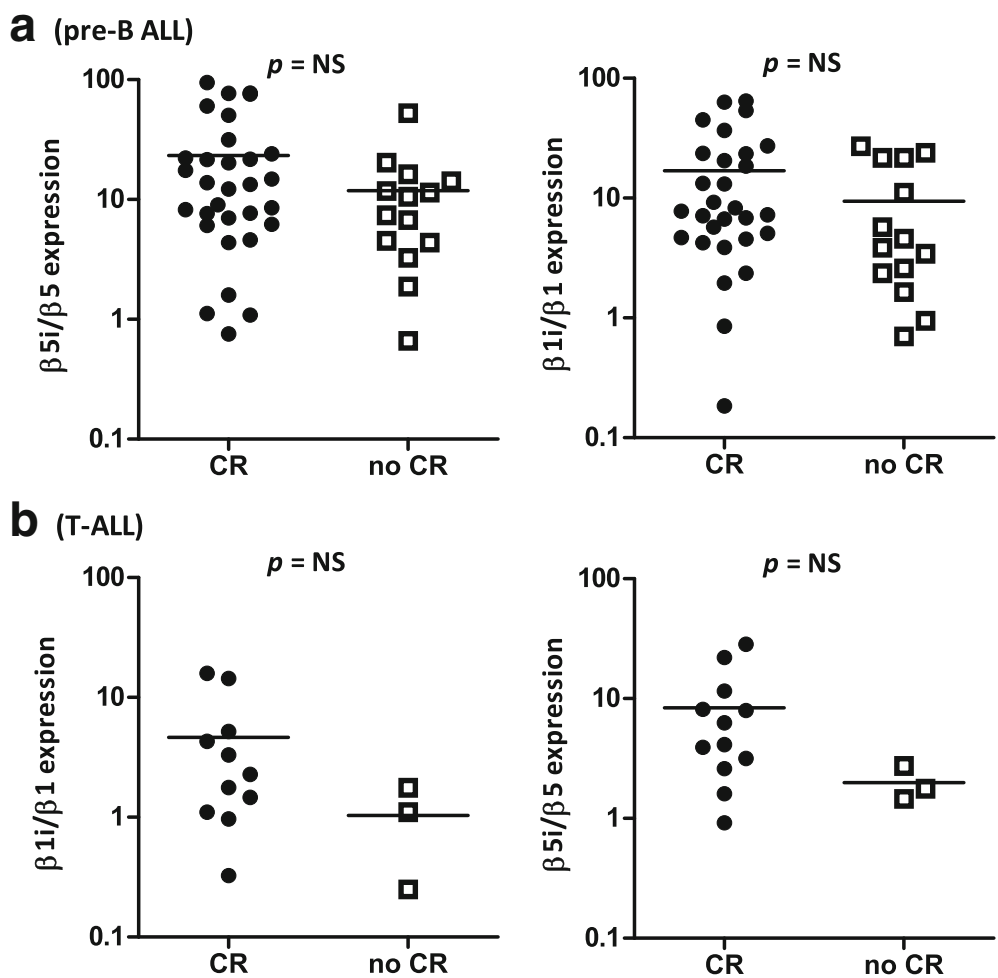

C (AML)
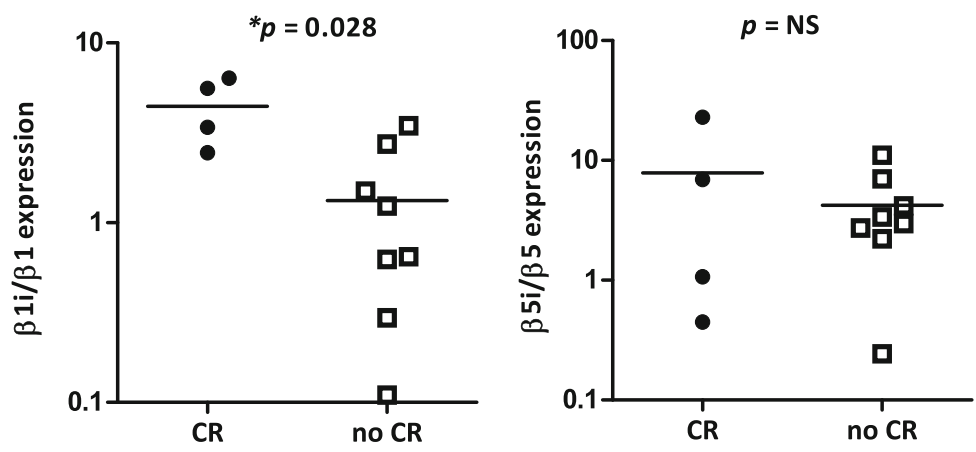

Fig. 2 Ratio proteasome subunit protein expression in patients who achieved complete remission versus patients who did not, dissected by leukaemia subtype. Ratios of immunoproteasome/constitutive proteasome protein expression in a AML patients, $\mathbf{b}$ pre-B ALL patients and c T-ALL patients that achieved CR versus patients who did not achieve CR, determined by Western blot analysis after bortezomibcontaining re-induction therapy. The line denotes the mean 
$\mathrm{CR}$ and patients that did not attain CR. Despite the fact that pre-treatment ratios of $\beta 5 \mathrm{i} / \beta 5$ or $\beta 1 \mathrm{i} / \beta 1$ were related to achievement of $C R$, these parameters were not strong enough to independently predict treatment response in either univariable logistic regression analyses or after controlling for blast percentage, leukaemia subtype, gender, age and white blood cell count in multivariable analysis (data not shown).

\section{Proteasome catalytic activity}

There was a good correlation between proteasome subunit expression and catalytic activity for $\beta 5 \mathrm{i} / \beta 5(R=0.503$ $p=0.005$, Fig. 3a). The median activity ratio of $\beta 5 \mathrm{i} / \beta 5$ differed significantly between pre-B ALL $(n=18)$ and AML $(n=10)$ patients $(p=0.005$, Fig. $3 \mathrm{~b})$ while the individual subunits $\beta 5$ and $\beta 5 \mathrm{i}$ did not differ (data not shown). In addition to proteasome subunit expression ratios, median $\beta 5 i / \beta 5$ subunit activity ratios were not higher in patients who reached CR $(n=14)$ compared to patients who did not reach CR $(n=18)$ (Fig. 3c). Median blast percentages did not differ statistically between CR patients (median $81 \%$ ) and no CR patients (median $71 \%$ ).

\section{The effect of bortezomib treatment on NF-kB activity}

We also examined NF-kB activity in leukaemia cells in response to bortezomib-containing chemotherapy. NF-kB activity was evaluated in PBMCs of 36 ALL patients (26 pre-B ALL and $10 \mathrm{~T}$-ALL) and 12 AML patients at three time points: $T=0, T=6$ and $T=24 \mathrm{~h}$ after the first bortezomib administration. Median pre-treatment NF-kB activity was significantly lower in pre-B ALL versus both TALL patients $(p=0.001)$ and AML patients $(p<0.001)$ (Fig. 4a, Table 2), while blast percentages were similar for these groups. Twenty-four hours after bortezomib treatment, pre-B ALL patients who attained a CR $(n=16)$ displayed a significant decrease in median NF-kB activity compared to pre-treatment levels $(p=0.006$, Fig. $4 \mathrm{~b})$. In contrast, pre-B ALL patients who did not attain a complete remission $(n=10)$ after the first bortezomib cycle showed unaltered NF-kB activity after bortezomib treatment (Fig. 4c). Overall, however, changes in NF-kB activity during treatment with bortezomib-containing chemotherapy were not significantly associated with achievement of post-re-induction CR.

\section{Discussion}

Recently, on the basis of an ex vivo study with acute leukaemia clinical specimen at diagnosis, we provided evidence that ratios of immunoproteasome subunits to constitutive subunits correlate with sensitivity of acute leukaemia cells to PIs [10]. The current research was set up to validate this in a distinct, prospectively collected sample set from patients enrolled on two COG clinical trials, which is the first to study potential biomarkers that may predict clinical sensitivity to bortezomibcontaining treatment regimens.

Although approved and efficacious for treatment of MM, bortezomib treatment does not lead to response in all MM patients due to emergence of drug resistance [24]. Conceivably, resistance phenomena could also limit the clinical utility of this agent in relapsed paediatric leukaemia. Hence, parameters that could indicate the likelihood of clinical response to PI therapy would provide a mechanism to determine which patients are likely to benefit from the addition of PI therapy to their reinduction regimen. Several mechanisms have been reported to underlie bortezomib resistance $[29,30]$. Bortezomib-resistant cell lines are frequently characterized by mutations in the PSMB5 gene encoding the $\beta 5$ subunit $[27,31]$. To date, however, no PSMB5 mutations have been found in patients clinically resistant to bortezomib [32-34]. Also in the current study, no PSMB5-
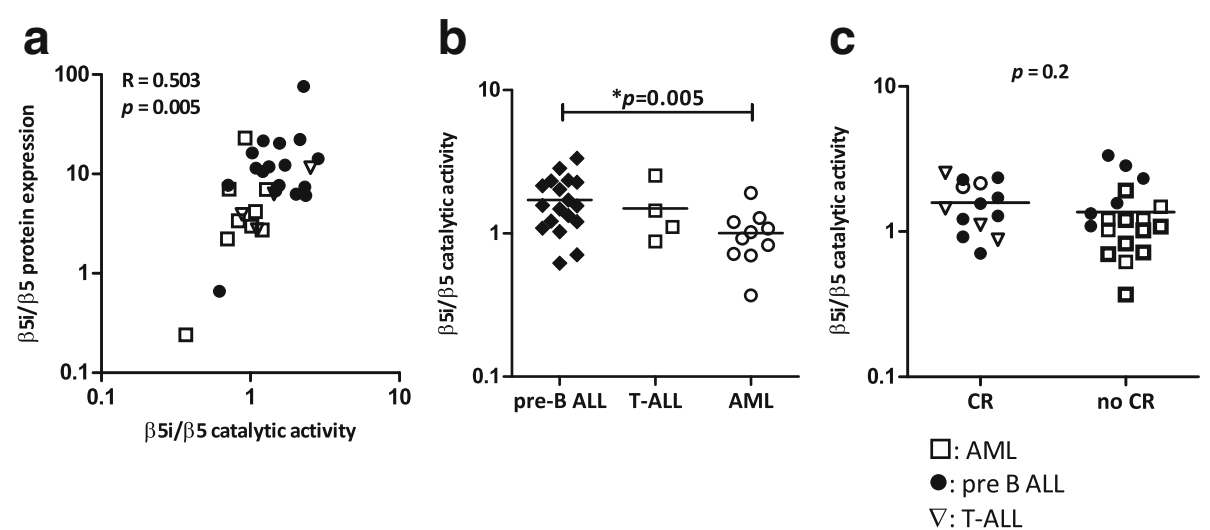

Fig. 3 Proteasome subunit catalytic activity in relapsed childhood ALL and AML patients. a Correlation of $\beta 5 i / \beta 5$ protein expression and $\beta 5 i / \beta 5$ catalytic activity ratios. b $\beta 5$ i/ $\beta 5$ catalytic activity ratios in pre-B ALL patients $(n=18)$ compared to T-ALL patients $(n=4)$ and AML patients $(n=$ 10), and $\mathbf{c} \beta 5 i / \beta 5$ catalytic activity ratios in patients who achieved complete remission $(n=14)$ versus those who did not $(n=18)$. Closed circles represent pre-B ALL patients, open triangles T-ALL patients and open squares AML patients. The line denotes the mean 

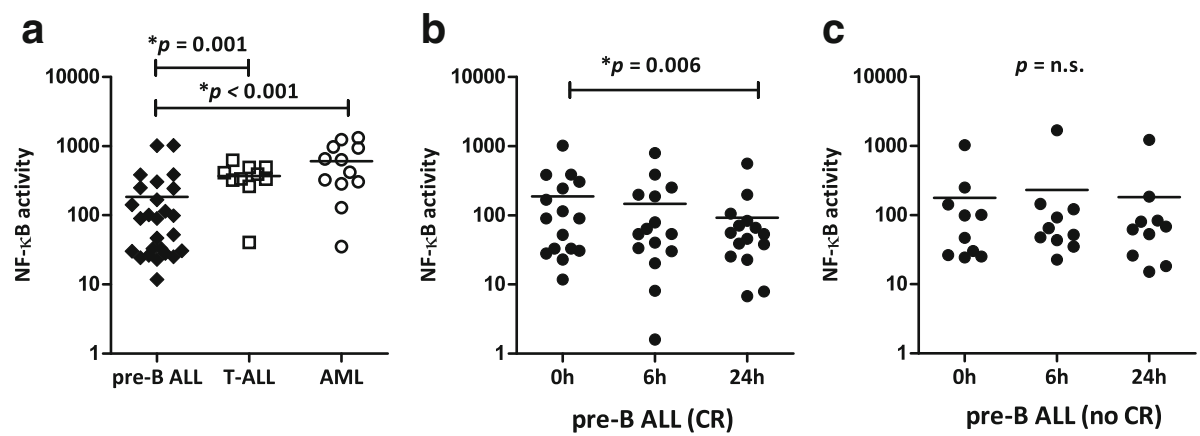

Fig. 4 Baseline NF-kB activity between ALL and AML patients and impact of bortezomib treatment. Pre-treatment NF-kB activity measurements determined by p65-ELISA in PBMC of acute leukaemia patients comparing a NF-kB activity of pre-B ALL $(n=26)$, T-ALL $(n=10)$ and $A M L(n=12)$ patients, and $\mathbf{b}$ NF-kB activity prior to treatment compared to 6 and $24 \mathrm{~h}$ after bortezomib-containing chemotherapy in pre-B ALL patients that reached CR $(n=16)$, and $\mathbf{c}$ pre-B ALL patients that did not reach CR $(n=10)$. The line denotes the mean

associated mutations in exon 2 of the gene were identified in either end of induction $(n=15)$ or relapse $(n=3)$ samples (data not shown). This implies that, in acute leukaemia, $\beta 5$ mutations make only a minor contribution to bortezomib resistance and that other common resistance mechanisms, such as overexpression of $\beta 5$, play a more important role [27, 29, 31, 35]. Confirming our earlier ex vivo work in newly diagnosed patient samples [10], the current study shows that constitutive ( $\beta 5$ and $\beta 1$ ) proteasome subunit expression was significantly lower in patients with relapsed ALL vs. AML, whereas $\beta 1 \mathrm{i}$ and $\beta 5 \mathrm{i}$ immunoproteasome subunit expression was higher (though it did not reach significance). These results were recently confirmed by de Bruin et al. [36], who showed by labelling analysis of primary patient cells that B cell ALL patients have higher immune/constitutive subunit expression ratios than AML cells and TALL cells. Given the significance of correlation between the subunit expression and catalytic activity (Fig. 3a), the subunit activity-based probes may prove useful for examining clinical specimen. Others have also reported that bortezomib sensitivity relates to proteasome expression. In particular, increased PSMB5 mRNA expression was found in a myeloma patient who subsequently developed bortezomib resistance [33]. Moreover, bortezomib-sensitive hematologic cell lines harboured higher immunoproteasome expression levels compared to relatively bortezomib-resistant solid-tumour cell lines [37]. Lastly, a higher $\beta 2 / \beta 1+\beta 5$ activity ratio correlated with higher bortezomib sensitivity in a cell line panel of hematologic malignancies [38]. In a recent study, we showed that interferon- $\gamma$-induced upregulation of immunoproteasome expression and concurrent constitutive proteasome subunit downregulation in bortezomibresistant hematologic tumour cell lines resulted in the sensitization for PI treatment [26]. Consistently, knockdown of constitutive $\beta 5$ in the AML cell line THP1 resulted in increased sensitivity to bortezomib [31].
NF-kB activity was evaluated by ELISA in 26 pre-B ALL, 10 T-ALL and 12 AML samples after the first dose of bortezomib. NF-kB is constitutively active in the majority of ALL patients [23, 39] and AML patients [40]. Though a significant decline in NF-kB activity was observed in pre-B ALL patients $24 \mathrm{~h}$ after the first bortezomib dose, this decline in NF-kB activity did not correlate with bortezomib response. Of note, Magrangeas et al. [41] found that low pretreatment levels of NF-kB were associated with a higher response rate to bortezomib-based induction in newly diagnosed MM, but we were unable to find a similar correlation between pre-treatment NF-kB activity and bortezomib. Overall, in our current study, changes in NF-kB activity during treatment with bortezomib-containing chemotherapy were not associated with achievement of post-induction $\mathrm{CR}$, and neither pre-treatment NF-kB activity nor decreases in NF-kB activity after $24 \mathrm{~h}$ correlated with clinical response.

This current cohort provided the first set of samples collected from patients who received bortezomib treatment and could serve as a potential validation cohort for our former test cohort [10]. Since the design of this add-on study was not yet optimized for the proteasome-based assays, there are some limitations that have to be considered. First, although only samples that contained $>20 \%$ of blasts were used, there was a large variation in blast percentage between samples (mean $72 \% \pm 23 \%$ ). To address the impact of normal cells, we measured proteasome subunit expression levels in normal PBMCs and found that these had a relative minor contribution on proteasome subunit expression in leukaemic cells and thus did not largely influence our analyses. Notwithstanding this fact, purification of lymphoblast/ myeloblast purification is recommended in future follow-up studies. Another important issue is the fact 
that patients in this study were treated with combination therapy. Hence, any outcome variable is a cumulative effect of all drugs, which can confound the relation between our measurements and bortezomib sensitivity.

\section{Conclusions}

In summary, cells of relapsed ALL patients had significantly higher $\beta 5 \mathrm{i} / \beta 5$ and $\beta 1 \mathrm{i} / \beta 1$ proteasome subunit protein expression ratios, and lower $\beta 5$ and $\beta 1$ constitutive subunit expression compared to cells of relapsed AML patients. Interestingly, AML patients who achieved CR after bortezomib-containing therapy had higher pretreatment immunoproteasome/constitutive proteasome expression ratios compared to patients who did not achieve CR. Further studies using purified blast populations are being conducted to confirm that higher ratios of immuno-/constitutive proteasome in pre-treatment ALL and AML cells are associated with an initial clinical response to bortezomib-containing re-induction treatment.

\section{Additional file}

Additional file 1: Figure S1. Individual immunoproteasome subunit protein expression in relapsed childhood acute leukaemia patients. Figure S2. Proteasome subunit mRNA expression in NBM cells. Figure S3. Proteasome subunit catalytic activity in relapsed childhood ALL and AML patients.

\section{Acknowledgements}

Not applicable.

\section{Funding}

This study was sponsored by KiKa (Children Cancer-free, GJLK), Fonds Stimulans (JC, SZ, GJ), U10CA098543 (Chairs Grant) and U10CA098413 (Statistics and Data Management) to the Children's Oncology group, an $\mathrm{NIH}-\mathrm{NCl}-\mathrm{K} 23$ (NIH-NCl-CA113775) (TMH), and grants from the Ladies Leukemia League and Takeda/Millennium Pharmaceuticals (TMH).

\section{Availability of data and materials}

The datasets (laboratory and patient data) supporting the conclusions of this article are included within the article (and its Additional file).

\section{Authors' contributions}

DN, JM and GaJ performed experiments; DN, GeJ, TMH, JC and PMV analysed the results; DN, GeJ, TMH and JC designed the research and wrote the paper. SZ and GJLK discussed the format and content of the article and contributed to the review and editing of the final manuscript. JAW, SPH, XL, TA and TMH provided study material and supervised the study. All authors agree with the content of this paper.

\section{Competing interests}

GJLK has been a member of a scientific advisory board concerning bortezomib in paediatric ALL for Johnson \& Johnson, more than 2 years ago. $\mathrm{TMH}$ received research funding from Takeda/Millennium Pharmaceuticals. All other authors declare no competing financial interests.

\section{Consent for publication}

Not applicable.

\section{Ethics approval and consent to participate}

Informed consent was obtained from the patient or their parent(s) and the studies have been performed according to the Declaration of Helsinki.
Assent, as appropriate, were obtained in accordance with the US National Cancer Institute, and the study was approved by the relevant COG committees, CTEP and the paediatric central IRB in accordance with institutional policies for human subjects' research. National Institutes of Health Reference Number: PAALL07P1\#A05PAMDREWW01.

\section{Author details}

'Department of Pediatric Oncology/Hematology, VU University Medical Center, Amsterdam, The Netherlands. ${ }^{2}$ Department of Amsterdam Rheumatology \& Immunology Center, VU University Medical Center, Amsterdam, The Netherlands. ${ }^{3}$ Department of Hematology, VU University Medical Center, De Boelelaan 1117, 1081 HV Amsterdam, The Netherlands. ${ }^{4}$ Department of Pediatrics, Texas Children's Cancer and Hematology Centers, Baylor College of Medicine, Houston, TX, USA. ${ }^{5}$ Department of Pediatrics, Hospital for Sick Children, University of Toronto, Toronto, Canada.

${ }^{6}$ Department of Pediatrics, University of Colorado Health Sciences Center, Denver, CO, USA. ${ }^{7}$ COG Operations Office, Arcadia, CA, USA. ${ }^{8}$ Department of Preventive Medicine, University of Southern California, Los Angeles, CA, USA. ${ }^{9}$ Department of Epidemiology and Biostatistics, VU University Medical Center, Amsterdam, The Netherlands.

Received: 15 July 2016 Accepted: 25 August 2016

Published online: 06 September 2016

\section{References}

1. Pui $\mathrm{CH}$, Mullighan $\mathrm{CG}$, Evans WE, Relling MV. Pediatric acute lymphoblastic leukemia: where are we going and how do we get there? Blood. 2012;120:1165-74.

2. Kaspers GJ. Pediatric acute myeloid leukemia. Expert Rev Anticancer Ther. 2012;12:405-13.

3. Moreau P, Richardson PG, Cavo M, Orlowski RZ, San Miguel JF, Palumbo A, et al. Proteasome inhibitors in multiple myeloma: 10 years later. Blood. 2012;120:947-59.

4. Fisher RI, Bernstein SH, Kahl BS, Djulbegovic B, Robertson MJ, de Vos S, et al. Multicenter phase II study of bortezomib in patients with relapsed or refractory mantle cell lymphoma. J Clin Oncol. 2006;24:4867-74.

5. Horton TM, Gannavarapu A, Blaney SM, D'Argenio DZ, Plon SE, Berg SL. Bortezomib interactions with chemotherapy agents in acute leukemia in vitro. Cancer Chemother Pharmacol. 2006:58:13-23.

6. Houghton PJ, Morton CL, Kolb EA, Lock R, Carol H, Reynolds CP, et al. Initial testing (stage 1 ) of the proteasome inhibitor bortezomib by the pediatric preclinical testing program. Pediatr Blood Cancer. 2008;50:37-45.

7. Horton TM, Perentesis JP, Gamis AS, Alonzo TA, Gerbing RB, Ballard J, et al. A Phase 2 study of bortezomib combined with either idarubicin/cytarabine or cytarabine/etoposide in children with relapsed, refractory or secondary acute myeloid leukemia : a report from the children's oncology group. Pediatr Blood Cancer. 2014;61:1754-60.

8. Messinger $\mathrm{YH}$, Gaynon PS, Sposto R, van der GJ, Eckroth E, Malvar J, et al. Bortezomib with chemotherapy is highly active in advanced B-precursor acute lymphoblastic leukemia: Therapeutic Advances in Childhood Leukemia \& Lymphoma (TACL) Study. Blood. 2012;120:285-90.

9. Groettrup M, Kirk CJ, Basler M. Proteasomes in immune cells: more than peptide producers? Nat Rev Immunol. 2010;10:73-8.

10. Niewerth D, Franke NE, Jansen G, Assaraf YG, van Meerloo J, Kirk CJ, et al. Higher ratio immune vs. constitutive proteasome level as novel indicator of sensitivity of pediatric acute leukemia cells to proteasome inhibitors. Haematologica. 2013;98:1896-904.

11. Parlati F, Lee SJ, Aujay M, Suzuki E, Levitsky K, Lorens JB, et al. Carfilzomib can induce tumor cell death through selective inhibition of the chymotrypsin-like activity of the proteasome. Blood. 2009;114:3439-47.

12. Basler M, Kirk CJ, Groettrup M. The immunoproteasome in antigen processing and other immunological functions. Curr Opin Immunol. 2012;25:74-80.

13. Obeng EA, Carlson LM, Gutman DM, Harrington WJ, Lee KP, Boise LH. Proteasome inhibitors induce a terminal unfolded protein response in multiple myeloma cells. Blood. 2006;107:4907-16.

14. McConkey DJ, Zhu K. Mechanisms of proteasome inhibitor action and resistance in cancer. Drug Resist. 2008;11:164-79.

15. Horton TM, Pati D, Plon SE, Thompson PA, Bomgaars LR, Adamson PC, et al. A phase 1 study of the proteasome inhibitor bortezomib in pediatric 
patients with refractory leukemia: a Children's Oncology Group study. Clin Cancer Res. 2007;13:1516-22.

16. Cortes J, Thomas D, Koller C, Giles F, Estey E, Faderl S, et al. Phase I study of bortezomib in refractory or relapsed acute leukemias. Clin Cancer Res. 2004;10:3371-6.

17. Faderl S, Rai K, Gribben J, Byrd JC, Flinn IW, O'Brien S, et al. Phase II study of single-agent bortezomib for the treatment of patients with fludarabinerefractory B-cell chronic lymphocytic leukemia. Cancer. 2006;107:916-24.

18. Santos FP, Kantarjian H, McConkey D, O'Brien S, Faderl S, Borthakur G, et al. Pilot study of bortezomib for patients with imatinib-refractory chronic myeloid leukemia in chronic or accelerated phase. Clin Lymphoma Myeloma Leuk. 2011;11:355-60.

19. Minderman H, Zhou Y, O'Loughlin KL, Baer MR. Bortezomib activity and in vitro interactions with anthracyclines and cytarabine in acute myeloid leukemia cells are independent of multidrug resistance mechanisms and p53 status. Cancer Chemother Pharmacol. 2007;60:245-55.

20. Attar EC, De Angelo DJ, Supko JG, D'Amato F, Zahrieh D, Sirulnik A, et al. Phase I and pharmacokinetic study of bortezomib in combination with idarubicin and cytarabine in patients with acute myelogenous leukemia. Clin Cancer Res. 2008;14:1446-54.

21. Blum W, Schwind S, Tarighat SS, Geyer S, Eisfeld AK, Whitman S, et al. Clinical and pharmacodynamic activity of bortezomib and decitabine in acute myeloid leukemia. Blood. 2012;119:6025-31.

22. Orlowski RZ, Voorhees PM, Garcia RA, Hall MD, Kudrik FJ, Allred T, et al. Phase 1 trial of the proteasome inhibitor bortezomib and pegylated liposomal doxorubicin in patients with advanced hematologic malignancies. Blood. 2005;105:3058-65.

23. Brown RE, Bostrom B, Zhang PL. Morphoproteomics and bortezomib/ dexamethasone-induced response in relapsed acute lymphoblastic leukemia. Ann Clin Lab Sci. 2004;34:203-5.

24. Kumar SK, Lee JH, Lahuerta JJ, Morgan G, Richardson PG, Crowley J, et al. Risk of progression and survival in multiple myeloma relapsing after therapy with IMiDs and bortezomib: a multicenter international myeloma working group study. Leukemia. 2012;26:149-57.

25. Niewerth D, Jansen G, Assaraf YG, Zweegman S, Kaspers GJL, Cloos J. Molecular basis of resistance to proteasome inhibitors in hematological malignancies. Drug Resist Updat. 2015;18:18-35.

26. Niewerth D, Kaspers GJL, Assaraf YG, van Meerloo J, Kirk CJ, Anderl JA, et al. Interferon-y-induced upregulation of immunoproteasome subunit assembly overcomes bortezomib resistance in human hematological cell lines. J Hematol Oncol. 2014;7.

27. Franke NE, Niewerth $D$, Assaraf $Y G$, Van Meerloo J, Vojtekova K, van Zantwijk $\mathrm{CH}$, et al. Impaired bortezomib binding to mutant beta5 subunit of the proteasome is the underlying basis for bortezomib resistance in leukemia cells. Leukemia. 2012;26:757-68.

28. Blackburn C, Gigstad KM, Hales P, Garcia K, Jones M, Bruzzese FJ, et al. Characterization of a new series of non-covalent proteasome inhibitors with exquisite potency and selectivity for the 205 beta5-subunit. Biochem J. 2010;430:461-76.

29 Cloos J, Niewerth D, Jansen G. Pre-clinical studies on the molecular basis of bortezomib resistance and modalities to overcome resistance in hematological malignancies. In: Dou QP, ed. Resist. to proteasome Inhib. cancer. Switzerland: Springer International Publishing Switzerland; 2014. p. $181-204$.

30 Cottini F, Guidetti A, Paba Prada C, Hideshima T, Maglio M, Varga D, et al. Resistance to proteasome inhibitors in multiple myeloma. In: Dou QP, ed. Resist. to Proteasome Inhib. Cancer. Switzerland: Springer International Publishing Switzerland; 2014. p. 47-80.

31 Oerlemans R, Franke NE, Assaraf YG, Cloos J, van Zantwijk I, Berkers CR, et al. Molecular basis of bortezomib resistance: proteasome subunit beta5 (PSMB5) gene mutation and overexpression of PSMB5 protein. Blood. 2008:112:2489-99.

32 Politou M, Karadimitris A, Terpos E, Kotsianidis I, Apperley JF, Rahemtulla A. No evidence of mutations of the PSMB5 (beta- 5 subunit of proteasome) in a case of myeloma with clinical resistance to Bortezomib. Leuk Res. 2006;30:240-1.

33 Shuqing L, Jianmin Y, Chongmei H, Hui C, Wang J. Upregulated expression of the PSMB5 gene may contribute to drug resistance in patient with multiple myeloma when treated with bortezomib-based regimen. Exp Hematol. 2011:39:1117-8.
34 Lichter DI, Danaee H, Pickard MD, Tayber O, Sintchak M, Shi H, et al. Sequence analysis of $\beta$-subunit genes of the $20 \mathrm{~S}$ proteasome in patients with relapsed multiple myeloma treated with bortezomib or dexamethasone. Blood. 2012:120:4513-6.

35 Lu S, Chen Z, Yang J, Chen L, Gong S, Zhou H, et al. Overexpression of the PSMB5 gene contributes to bortezomib resistance in Tlymphoblastic lymphoma/leukemia cells derived from Jurkat line. Exp Hematol. 2008;36:1278-84.

36 de Bruin G, Xin BT, Kraus M, van der Stelt M, van der Marel GA, Kisselev AF, et al. A set of activity-based probes to visualize human (immuno)proteasome activities. Angew Chem Int Ed Engl. 2016;55:4199-203.

37 Busse A, Kraus M, Na IK, Rietz A, Scheibenbogen C, Driessen C, et al. Sensitivity of tumor cells to proteasome inhibitors is associated with expression levels and composition of proteasome subunits. Cancer. 2008:112:659-70.

38 Kraus M, Ruckrich T, Reich M, Gogel J, Beck A, Kammer W, et al. Activity patterns of proteasome subunits reflect bortezomib sensitivity of hematologic malignancies and are variable in primary human leukemia cells. Leukemia. 2007;21:84-92.

39 Kordes U, Krappmann D, Heissmeyer V, Ludwig WD, Scheidereit C. Transcription factor NF-kappaB is constitutively activated in acute lymphoblastic leukemia cells. Leukemia. 2000;14:399-402.

40 Guzman ML, Neering SJ, Upchurch D, Grimes B, Howard DS, Rizzieri DA, et al. Nuclear factor-kappaB is constitutively activated in primitive human acute myelogenous leukemia cells. Blood. 2001;98:2301-7.

41 Magrangeas F, Moreau P, Campion L, Avet-Loiseau H, Guérin C, Gouraud W, et al. Low level of NF-Kb activity is associated with higher response rate to bortezomib-based induction therapy in patients with newly diagnosed multiple myeloma. ASH Annu Meet Abstr. 2013;122:3106.

\section{Submit your next manuscript to BioMed Central and we will help you at every step:}

- We accept pre-submission inquiries

- Our selector tool helps you to find the most relevant journal

- We provide round the clock customer support

- Convenient online submission

- Thorough peer review

- Inclusion in PubMed and all major indexing services

- Maximum visibility for your research

Submit your manuscript at www.biomedcentral.com/submit
Biomed Central 